\title{
MULTI-SATELLITE SCHEDULING APPROACH FOR DYNAMIC AREAL TASKS TRIGGERED BY EMERGENT DISASTERS
}

\author{
X. N. Niu ${ }^{\text {a }}$ X. J. Zhai ${ }^{\text {a }}$ H. Tang ${ }^{\text {a, }}$ *, L. X. Wu ${ }^{\text {b }}$ \\ a State Key Laboratory of Earth Surface Processes and Resource Ecology, Beijing Normal University, 19 Xinjiekouwai Street \\ Beijing, China - (niuxiaonan, zhai.xuejun)@mail.bnu.edu.cn, hongtang@bnu.edu.cn \\ ${ }^{\mathrm{b}}$ School of Environment Science and Spatial Informatics, China University of Mining and Technology, Xuzhou 221116, China - \\ awulixin@263.net
}

\section{WG I/V}

KEY WORDS: Satellite Mission Scheduling, Emergent Disasters, Satellite Scheduling Model, Dynamic Segmenting Algorithm, Heuristic algorithm, Greedy criterion, Wenchuan Earthquake

\begin{abstract}
:
The process of satellite mission scheduling, which plays a significant role in rapid response to emergent disasters, e.g. earthquake, is used to allocate the observation resources and execution time to a series of imaging tasks by maximizing one or more objectives while satisfying certain given constraints. In practice, the information obtained of disaster situation changes dynamically, which accordingly leads to the dynamic imaging requirement of users. We propose a satellite scheduling model to address dynamic imaging tasks triggered by emergent disasters. The goal of proposed model is to meet the emergency response requirements so as to make an imaging plan to acquire rapid and effective information of affected area. In the model, the reward of the schedule is maximized. To solve the model, we firstly present a dynamic segmenting algorithm to partition area targets. Then the dynamic heuristic algorithm embedding in a greedy criterion is designed to obtain the optimal solution. To evaluate the model, we conduct experimental simulations in the scene of Wenchuan Earthquake. The results show that the simulated imaging plan can schedule satellites to observe a wider scope of target area. We conclude that our satellite scheduling model can optimize the usage of satellite resources so as to obtain images in disaster response in a more timely and efficient manner.
\end{abstract}

\section{INTRODUCTION}

Natural disasters break out frequently in the earth, which causes serious environmental and economic losses. Since all kinds of natural disasters (earthquakes, landslides, floods, hurricanes, debris flow, etc.) often happen unexpectedly and destructively, it bring great difficulties for emergency disaster relief work. As is known, remote sensing technology plays a very important role in natural disaster reduction. The earth observation satellite can be used to perform a wide range of observation activity to obtain the information of disaster formation, development and dynamic change, which can provide data of disaster monitoring timely. With the development of satellite application technology, more and more imaging satellite have been launched into space. However, satellite resources are still scarce with respect to the increasing human demands for imaging. In order to optimize the usage of remote sensing satellite resources as well as obtaining high-quality images in disaster response in a more timely and efficient manner, satellite mission scheduling should be used.

The process of satellite mission scheduling is used to allocate the observation resources, i.e., the sensors of the satellites and execution time to a series of imaging tasks by maximizing one or more objectives while satisfying certain given constraints. According to the area of observation targets, the research on satellite mission scheduling can be divided into two classes: satellite scheduling problem of point target and satellite scheduling problem of area target. Over the last several decades, development of methods to perform satellite mission scheduling has been intensively investigated, most of which are focused on the point target. In order to facilitate solving the satellite scheduling problem which is very complex, most studies established the corresponding mathematical model based on the practical application. Such models mainly included mathematical programming model (Bensana et al., 1996), constraint satisfaction model (Pemberton, 2000), graph model (Gabrel and Vanderpooten, 2002). Moreover, to solve the model, the researchers presented many algorithms, most of which were approximate methods. These approximate methods mainly included intelligent optimization algorithms and rulebased heuristic algorithms. The intelligent optimization algorithms, such as the Tabu search algorithm (Bianchessi et al., 2007), the genetic algorithm (Mansour and Dessouky, 2010; Niu et al., 2015), the evolutionary algorithm (Wang et al., 2007), simulated annealing, the Lagrangian relaxation technique (Lin et al., 2005), and the hybrid ant colony optimization method (Wu et al., 2013; Zhang et al., 2014), can be used to obtain near-optimal solutions for large size problems. In addition, rulebased heuristic algorithms were also designed to solve the satellite scheduling (Hall and Magazine, 1994; Wang et al., 2011; Zhai et al., 2015). The rule-based heuristic methods are more flexible to obtain satisfactory solutions. To summarize, these methods can provide near-optimal solutions to largescaled problems.

Up to now, there have been a few researches towards the satellite scheduling problem for area target. Some methods

* Corresponding author 
(Walton, 1993; Lemaître and Verfaillie, 1997; Lemaître et al., 2002; Mancel 2003; Lin et al., 2005; Li et al., 2008; Li et al., 2009) can only solve the scheduling problem for single satellite or single area target. Focus on multi-satellite and multi-target scheduling problem, Ruan et al. (2006) presented a method on segmenting area target based on the characteristics of the sensor and target. Bai et al. (2009) further improved the algorithm and proposed two optimization algorithm. However, all studies mentioned above did not take into account the dynamic imaging requirement. According to the actual requirement, users may submit some new tasks when a scheduling scheme is being executed. Especially, there are some unexpectedly coming tasks caused by emergent event. Unfortunately, to the best of our knowledge, little attention is paid to satellite task scheduling for dynamic imaging demands triggered by emergent disasters.

In the paper, we focus on the dynamic areal tasks triggered by emergent disasters. Once natural disasters occur, there is an urgent need to reasonably utilize the existing satellites to rapidly image the affected area. Actually the disaster information obtained changes dynamically, which accordingly leads to new imaging requirement of users. For example, the users will submit new tasks timely. In this paper, we propose a satellite scheduling model to address dynamic emergency tasks submitted timely. In the model, the reward of the schedule is maximized. A dynamic segmenting algorithm is designed to partition area targets in satellite scheduling. Then we present the dynamic heuristic algorithm embedding in a greedy criterion to obtain the optimal solution.

The remainder of the paper is organized as follows. In section 2, we state the scheduling problem for area task and describe the dynamic segmenting algorithm. Then we present the satellite scheduling model in section 3 . In section 4 , we propose a dynamic heuristic algorithm embedding in a greedy criterion to dynamically insert new imaging requirement. In section 5 , we conduct experimental simulations. We conclude the paper with a summary in section 6 .

\section{DYNAMIC SEGMENTING ALGORITHM}

In the satellite scheduling problem, according to the area of the targets, the imaging tasks can be divided into two types: point target and area target. The point target (e.g. house, bridge) is small so that it can be covered by a single observations trip. As for the area target, which covers a wide geographical area, it cannot be photographed in a scene. The area target may require several contiguous strips to be completely imaged. Usually, the area target can be segmented into several sub-targets to be imaged. As shown in Figure 1, the EOS operates in space in a certain orbit. When the EOS flies over the target area, its sensor is opened to take the image. As the imaging process will last a few seconds, it will produce a strip that covers the target. Since the point task such as $t_{1}$ is small so it can be covered by a single strip. While the area task like $t_{5}$ need several contiguous strips to be completely imaged as shown in Figure 2. The process of cutting-up the area target into contiguous strips is controlled by the direction and width of a single strip which depends on the field of view and the height of the satellite.

In the paper, we focus on the dynamic areal target triggered by emergent disasters. Due to the wide coverage of the disaster area, we describe it as an area target in our scheduling problem. For area target, segmenting is the first step before modelling. Therefore, we firstly design a dynamic segmenting algorithm to partition area targets. We use the segmentation results as input data of the model constructed later.

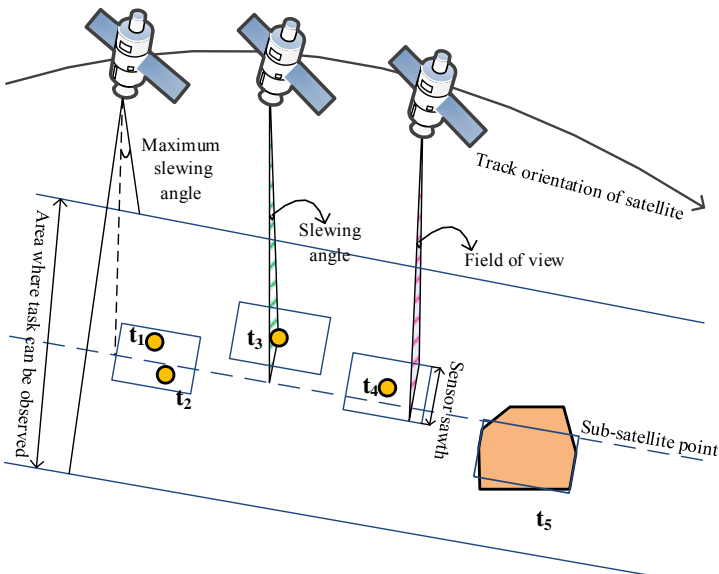

Figure 1. Illustration of satellite observing activity

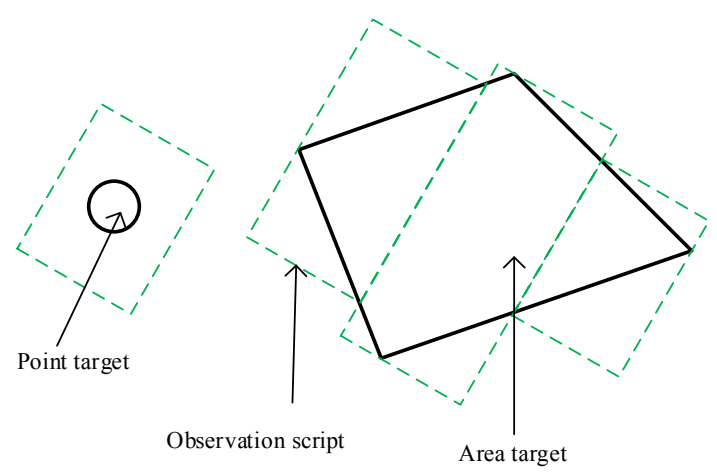

Figure 2. Illustration of the point target and area target

Assume there are a set of area tasks $R A=\left\{r a_{1}, \cdots, r a_{M}\right\}$ to be observed by satellites $S=\left\{s^{1}, s^{2}, \cdots, s^{J}\right\}$ where $J$ is the number of satellites. For a task $r a_{i} \in R A$, we define its time windows on satellite $j$ as a set $T W_{i}^{j}=\left\{t w_{i 1}^{j}, t w_{i 2}^{j}, \cdots, t w_{i K_{i j}}^{j}\right\}$ where $K_{i j}$ is the number of time windows for task $r a_{i}$ on satellite $s^{j}$.For each time window $t w_{i k}^{j}, t w_{i k}^{j}=\left[w s_{i k}^{j}, w e_{i k}^{j}\right]$. The dynamic segmenting method that we design is as follows:

Step1: For each task $r a_{i} \in R A$, select the available satellites $s^{j} \in S$ according to the requirements of users for resolution and so on.

Step2: For each satellite $s^{j} \in S$, compute the time windows $T W_{i}^{j}=\left\{t w_{i 1}^{j}, t w_{i 2}^{j}, \cdots, t w_{i K_{i j}}^{j}\right\}$ between $r a_{i}$ and $s^{j}$.

Step3: For each time window $t w_{i k}^{j} \in T W_{i}^{j}$, decompose the area task $r a_{i}$.

Step3.1: Within $t w_{i k}^{j}$, obtain the minimum and maximum observation angle $\theta_{\min }(i, j), \theta_{\max }(i, j)$.

Step3.2: Compute the effective observation angle taking the slewing ability of satellite into account: 


$$
\left\{\begin{array}{l}
\theta_{\text {min }}=\max \left(\theta_{\text {min }}(i, j)+\frac{1}{2} \Delta \theta^{j},-m s g^{j}\right) \\
\theta_{\text {max }}=\min \left(\theta_{\text {max }}(i, j)-\frac{1}{2} \Delta \theta^{j}, m s g^{j}\right)
\end{array}\right.
$$

where $\Delta \theta^{j}=$ the field of view

$m s g^{j}=$ the maximum swing angle of satellite $s^{j}$.

Step3.3: Use different observation angle to partition the area target. After setting the excursion angle parameter $\Delta \lambda$, obtain a set of observation angles $\theta(i, j)=\left\{\theta_{i k 1}^{j}, \theta_{i k 2}^{j}, \cdots, \theta_{i k V_{i j}}^{j}\right\}$ where $\theta_{i k 1}^{j}=\theta_{\min }, \theta_{i k v}^{j}=\theta_{i k(v-1)}^{j}+\Delta \lambda, \theta_{i k m}^{j}=\theta_{\max }$.

Step3.4: With regard to each observation angle, compute the bounds of strip that satellite can observe which we denote as a meta-task $r o_{i k v}^{j}$. As a result, create a set of meta-tasks $r o_{i k}^{j}=\left\{r o_{i k 1}^{j}, r o_{i k 2}^{j}, \cdots, r o_{i k V_{i j}}^{j}\right\}$.

Step3.5: For each meta-task $r o_{i k v}^{j}$, compute the time window $t w_{i k v}^{j}=\left[w s_{i k v}^{j}, w e_{i k v}^{j}\right]$ with the observation angle $\theta_{i k v}^{j}$ in the scope of $t w_{i k}^{j}$.

Step4: The meta-tasks decomposed by $r a_{i}$ within $T W_{i}^{j}$ is $r o_{i}^{j}=\bigcup_{k=1}^{K_{i j}} r o_{i k}^{j}$

Step5: Let the meta-tasks decomposed by $r a_{i}$ on all satellites $r o_{i}=\bigcup_{j=1}^{J} r o_{i}^{j}$.

Step6: If all tasks $r a_{i} \in R A$ are partitioned into meta-tasks, then output the results $r o$, for all $i \in\{1,2, \cdots, M\}$.

\section{MODEL}

The satellite scheduling problem usually consists of five parts: tasks, satellite resources, opportunities, objectives and constraints.

\subsection{Tasks}

In the paper, we focus on the area tasks. Let $R A=\left\{r a_{1}, \cdots, r a_{M}\right\}$ be the set of tasks to be observed where $M$ is the number of total area tasks. Each emergency task $r a_{i} \in R A$ has a weight $w_{i}$, an arrival time $a t_{i}$ and a deadline $d t_{i}$. Because the task must be segmented into strips that can be imaged once, we call these strips meta-tasks. In the scheduling model, the meta-task set are regarded as input. Let $r o_{i}=\bigcup_{j=1}^{J} r o_{i}^{j}$ be the meta-task set of $r a_{i}$ decomposed by segmenting method presented as above. Each meta-task $r o_{i k v}^{j} \in r o_{i}^{j}$ has an indispensable duration of task execution $d_{i k v}^{j}$.
The uncertainty of the arrival time of emergent tasks will trigger multiple dynamic scheduling during a scheduling period. In other words, the algorithm should be timely adjusted the current schedules of planed tasks for performing new arriving tasks. Let $T_{S}=\left\{t_{S}^{1}, \cdots, t_{S}^{e}, t_{S}^{f}\right\}$ be the scheduling time set where $t_{S}^{i}(i \geq 1)$ is the $i$ th dynamic scheduling time, $e$ is the total batch of emergency tasks and is $t_{S}^{f}$ the end time of scheduling.

\subsection{Satellite Resources}

With regard to the satellites to perform the imaging tasks, we use $S=\left\{s^{1}, \cdots s^{J}\right\}$ to denote them. We assume that the sensors of the satellites considered in our study are able to slew laterally. Each satellite $s^{j} \in S$ can be denoted by $s^{j}=\left(\Delta \theta^{j}, \Delta d^{j}, s l^{j}\right.$, $\left.s t^{j}, m s g^{j}, o r b^{j}, s u^{j}, s d^{j}, d u t y^{j}\right)$ to describe its observation capability where $\Delta \theta^{j}, \Delta d^{j}, s l^{j}, s t^{j}, m s g^{j}, o r b^{j}, s u^{j}, s d^{j}$, $d u t y^{j}$ are the field of view, the longest duration allowed for a continuous observation, slewing rate, attitude stability time, the maximum swing angle, the flight time in each orbit, the start-up time of sensor, the retention time of shutdown and the longest imaging time in each orbit, respectively.

\subsection{Time Windows}

A task must be performed by the satellites within the available time windows. For a meta-task $r o_{i k v}^{j}$, its corresponding the time window is $t w_{i k v}^{j}=\left[w s_{i k v}^{j}, w e_{i k v}^{j}\right]$ with the observation angle $\theta_{i k v}^{j}$.We define the time windows of $r a_{i}$ as a set $A O_{i}=\bigcup_{j=1}^{J} \bigcup_{k=1}^{K_{i j}} \bigcup_{v=1}^{V_{i j k}} a o_{i k v}^{j}$, where $a o_{i k v}^{j}=\left\{t w_{i k v}^{j}, \theta_{i k v}^{j}\right\}, V_{i j k}$ is the number of time windows between satellite $s^{j}$ and meta-tasks set $r o_{i k}^{j}, K_{i j}$ is the number of meta-tasks of $r a_{i}$ on satellite $s^{j}, J$ is the number of satellites.

\subsection{Objective}

We assume that $t_{s}^{k}$ is the current scheduling time. The primary objective is to maximize the reward of all observed target area. We think that the actual profit of a task $r a_{i}$ is linear with its observed area $\varphi\left(r a_{i}\right)$ :

$$
\max : \sum_{i=1}^{M} \operatorname{cov}_{i} \times w_{i}
$$

where $\operatorname{cov}_{i}=\frac{\left(\bigcup_{j=1}^{J} \bigcup_{k=1}^{K_{i j}} \bigcup_{v=1}^{V_{i j k}} x_{i k v}^{j} \varphi\left(r o_{i k v}^{j}\right)\right) \cap \varphi\left(r a_{i}\right)}{\varphi\left(r a_{i}\right)}$ is the proportion of acquired area of $r a_{i}$

$x_{i k v}^{j}$ is the decision variable that can be either 1 if the meta-task $r o_{i k v}^{j}$ is executed or 0 otherwise.

\subsection{Constraints}

In our model, regardless of the satellite measurement and control requirements and the data transmission with ground station requirements, there are four constraints that must be satisfied in the model. 
3.5.1 Time window constraint: If any meta-task is performed, then the imaging time must be within its time windows. Therefore, we have time window constraint

$$
\left\{\begin{array}{l}
x_{i k v}^{j}\left(t s_{i k v}^{j}-w e_{i k v}^{j}\right) \geq 0 \\
x_{i k v}^{j}\left(t s_{i k v}^{j}+d_{i k v}^{j}-w e_{i k v}^{j}\right) \leq 0 \\
a t_{i} \leq t_{s}^{k} \leq t s_{i k v}^{j} \leq d t_{i}
\end{array}\right.
$$

where $t s_{i k v}^{j}$ is the decision variable that denote the start time of the task $r o_{i k v}^{j}$.

3.5.2 Switch time constraint: In the scheduling scheme $S S=\bigcup_{j=1}^{J} S S^{j}$, where $S S^{j}$ is composed of a sequence of the scheduled tasks ordered in time on satellite $s^{j}$. There should be an adequate transition time between any two adjacent tasks $t_{m}, t_{n} \in S S^{j}$, for sensor to perform the actions including shutting down, swing the observation angle, stabilizing gesture and startup. Hence, we have the switch time constraint:

$$
t e_{m}^{j}+s d^{j}+t r_{m, n}^{j}+s u^{j} \leq t s_{n}^{j}
$$

where $\quad t e_{m}^{j}=$ the end time of task $t_{m}$

$\operatorname{tr}_{m, n}^{j}=s l^{j} \times\left|\theta_{m}^{j}-\theta_{n}^{j}\right|+s t^{j}$ denotes the transition time between task $t_{m}$ and $t_{n}$.

3.5.3 Task merging constraint: The tasks in the same swath of a sensor may be merged into one composite task. Given two tasks $r o_{i k v}^{j}$ and $r o_{i k u}^{j}$, they can be combined when the following conditions hold:

$$
\left\{\begin{array}{c}
\max \left\{w e_{i k v}^{j}, w e_{i k u}^{j}\right\}-\min \left\{w s_{i k v}^{j}, w s_{i k u}^{j}\right\} \leq \Delta d^{j} \\
\theta_{i k v}^{j}=\theta_{i k u}^{j}
\end{array}\right.
$$

Then the time window and the associated observation angle of the composite task $\mathrm{Cro}_{l}^{j}$ are

$$
\left\{\begin{array}{c}
W_{l}^{j}=\left[\min \left\{w s_{i k v}^{j}, w s_{i k u}^{j}\right\}, \max \left\{w e_{i k v}^{j}, w e_{i k v}^{j}\right\}\right] \\
\theta_{l}^{j}=\theta_{i k v}^{j}
\end{array}\right.
$$

3.5.4 Imaging time constraint: The total imaging time of any satellite $s^{j}$ should be less than the allowable longest imaging time during any orbit period time. Hereby, we have the imaging time constraint

$$
\sum_{i \in S S_{t_{b}}^{j}} d_{i} \leq d u t y^{j}
$$

where $S S_{t_{b}}^{j} \subseteq S S^{j}$ denotes a sequence of scheduled tasks on satellite $j$ which flies during the time $\operatorname{span} t_{b} \in\left[t_{b}, t_{b}+o r b^{j}\right]$, $t_{b} \in\left[t_{s}^{k}, t_{s}^{f}-o r b^{j}\right]$.

\section{ALGORITHM}

When the current schedule is executing, the users put forward new imaging requests. As a result, the new imaging tasks have to be scheduled. In this case, the current executing schedule must be modified to accommodate these new tasks. So it will result in disturbance to the schedule inevitably. Based on the current scheduling time, tasks may have different states: finished task, running task and waiting task. Only the sequence of waiting tasks can be inserted into new tasks. To arrange new coming tasks as more as possible in smaller disturbances and meet the timeliness requirement of the emergency tasks as well, we design a dynamic rule-based heuristic algorithm in which greedy criterion is embedded(GC-DAHA). The heuristic algorithm can produce satisfactory and feasible plans in a notably short time, which is suitable for large size problems. The greedy criterion acts as a role of heuristic rule, which is the basis and standard of decision-making.

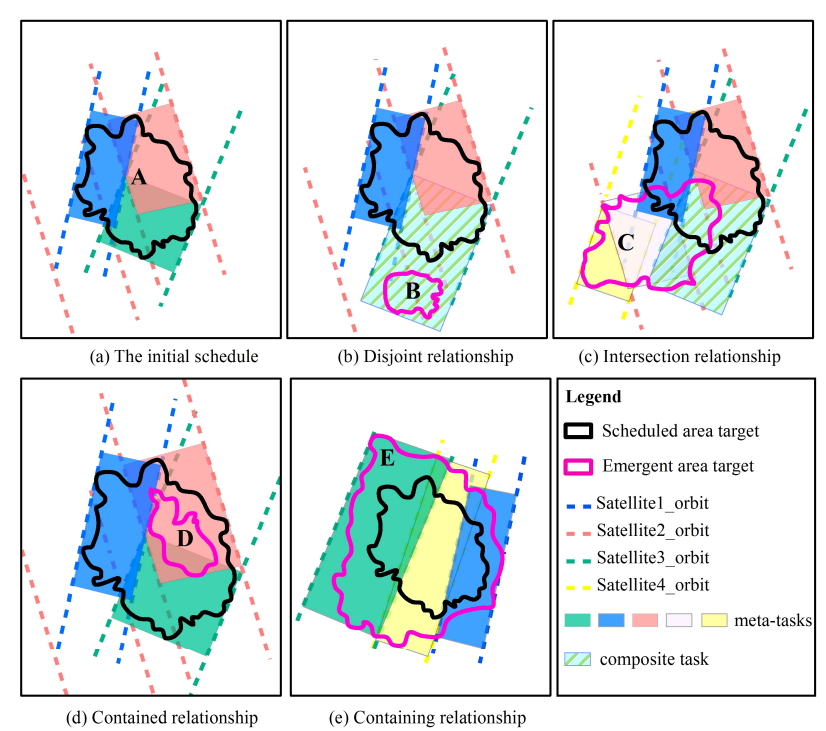

Figure 3. Spatial relationships between scheduled task and emergent task

Before the GC-DAHA algorithm, we pretreat each emergent area task firstly. By analyzing the spatial relationship between the task to schedule and the scheduled task in the waiting queue, we conclude that there exist three spatial relationships: disjoint, intersected, containing and contained. In Figure 3(a), we depict the current schedule for area task A. Suppose there comes an emergency task that we must insert to the waiting queue. If the emergent task B and scheduled task A are disjoint, as shown in Figure 3(b), we employ the GC-DAHA algorithm to insert the new emergent task. When the emergent task $\mathrm{C}$ intersects with the scheduled task, as shown in Figure 3(c), in the precondition that the two tasks have the same imaging requirement, we remove the intersection area from $\mathrm{C}$ and then schedule the remaining region of the task using the GC-DAHA algorithm. In Figure 3 (d), the emergent target is included in the scheduled task. If the former has higher weight, we must schedule the emergent task in priority. Then we schedule the remaining part of the scheduled task A. When the new target includes the scheduled task as shown in Figure 3 (e), we cancel the scheduled meta-tasks of target $\mathrm{A}$ and then schedule the new task using the GC-DAHA algorithm. 
The design of the GC-DAHA algorithm is described as Figure 4 In the GC-DAHA algorithm, each meta-task has three ways to be assigned into a scheduling sequence: insertion by task merging, direct insertion and insertion by deleting. Suppose there come $M$ dynamic area tasks to schedule. Decomposing them into meta-tasks by dynamic segmenting method, we add all meta-tasks $\bigcup_{i=1}^{M} r o_{i}$ into a queue $Q$. Then for all tasks in $Q$, we calculate the priority $p$ of a task according to the area coverage and weight: $p_{i k v}^{j}=\frac{\varphi\left(r o_{i k v}^{j}\right) \cap \varphi\left(r a_{i}\right)}{\varphi\left(r a_{i}\right)} \times w_{i}$. Set $p$ as the greedy criterion and select the task which has the maximum $p_{i k v}^{j}$ to be scheduled by three ways as mentioned above. For the task, we firstly judge whether it can merge with any other existing task in the current schedule. If the task fails to be merged, we attempt to insert the task directly if it does not conflict with any other task. If direct insertion fails, the conflict tasks will be deleted for inserting a new emergency task. The algorithm will terminate if the difference between the objectives which two times calculates is less than the threshold.

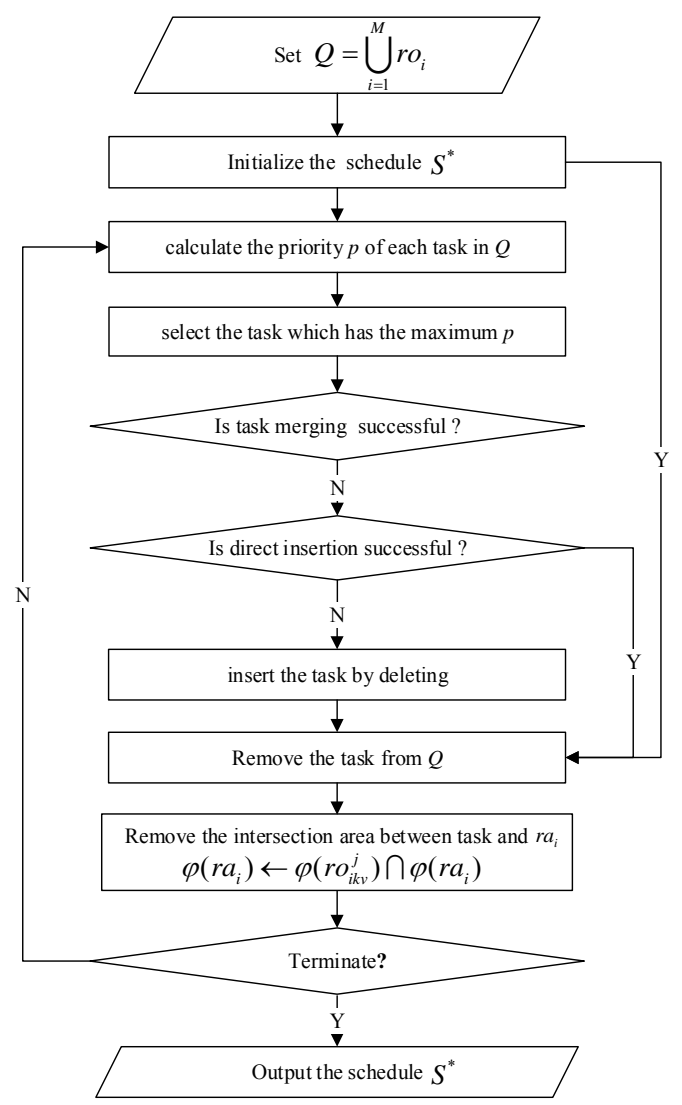

Figure 4. The algorithm of GC-DAHA

\section{EXPERIMENT}

We take Wenchuan Earthquake as the emergent event. After the earthquake occur, users will summit the imaging requirements. Based on the situation of disaster relief at that time, we simulate three batches of emergent tasks, which are located on 9 degree seismic intensity region, Wenchuan County, Beichuan County, respectively. As shown in Table 1, we present the information of tasks in detail. To complete the imaging tasks, we choose three satellites: GF-1, ZY1-02C, ZY-3. Each satellite circles the
Earth approximately 100 min each time. We set the scheduling period as three days.

\begin{tabular}{|l|c|c|c|l|}
\hline Task & Arrival time & Deadline & Weight & Location \\
\hline$r a_{1}$ & $\begin{array}{c}2008 / 05 / 12 \\
15: 00: 00\end{array}$ & $\begin{array}{c}2008 / 05 / 15 \\
15: 00: 00\end{array}$ & 10 & $\begin{array}{l}9 \text { degree } \\
\text { seismic } \\
\text { intensity } \\
\text { region }\end{array}$ \\
\hline$r a_{2}$ & $\begin{array}{c}2008 / 05 / 13 \\
00: 00: 00\end{array}$ & $\begin{array}{c}2008 / 05 / 14 \\
00: 00: 00\end{array}$ & 12 & $\begin{array}{l}\text { Wenchuan } \\
\text { County }\end{array}$ \\
\hline$r a_{3}$ & $\begin{array}{c}2008 / 05 / 14 \\
00: 00: 00\end{array}$ & $\begin{array}{c}2008 / 05 / 15 \\
00: 00: 00\end{array}$ & 14 & $\begin{array}{l}\text { Beichuan } \\
\text { County }\end{array}$ \\
\hline
\end{tabular}

Table 1. The information about emergent tasks

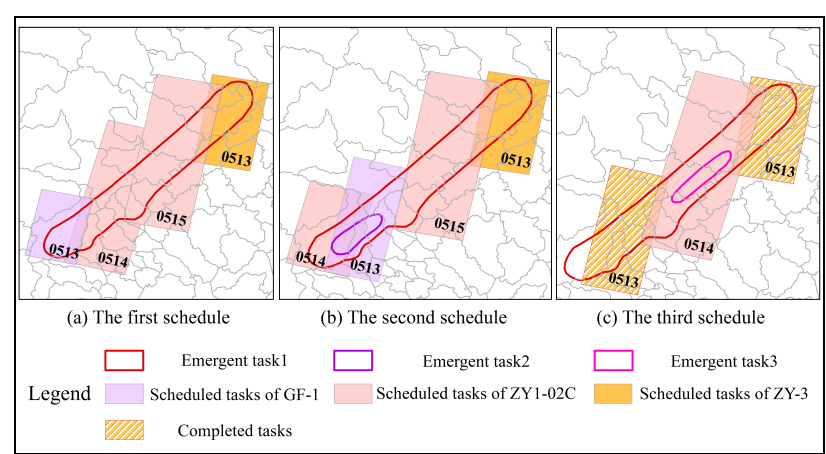

Figure 5. The simulated result

\begin{tabular}{|c|c|c|c|c|}
\hline $\begin{array}{c}\text { Meta } \\
\text { task }\end{array}$ & $\begin{array}{c}\text { Assigned } \\
\text { satellite }\end{array}$ & Start time & Duration/s & $\begin{array}{c}\text { Slewing } \\
\text { angle } /{ }^{\circ}\end{array}$ \\
\hline 1 & GF-1 & $\begin{array}{c}05 / 13 \\
10: 12: 09\end{array}$ & 12 & 2.5 \\
\hline 2 & ZY3 & $\begin{array}{c}05 / 13 \\
15: 31: 04\end{array}$ & 16 & 25 \\
\hline 3 & $\begin{array}{c}\text { ZY1- } \\
02 \mathrm{C}\end{array}$ & $\begin{array}{c}05 / 14 \\
12: 43: 34\end{array}$ & 25 & -22.5 \\
\hline 4 & $\begin{array}{c}\text { ZY1- } \\
02 \mathrm{C}\end{array}$ & $\begin{array}{c}05 / 15 \\
12: 11: 12\end{array}$ & 29 & 25 \\
\hline
\end{tabular}

Table 2. The schedule $S_{1}^{*}$

\begin{tabular}{|c|c|c|c|c|}
\hline $\begin{array}{c}\text { Meta } \\
\text { task }\end{array}$ & $\begin{array}{c}\text { Assigned } \\
\text { satellite }\end{array}$ & Start time & Duration/s & $\begin{array}{c}\text { Slewing } \\
\text { angle }^{\circ}\end{array}$ \\
\hline 1 & GF-1 & $\begin{array}{c}05 / 13 \\
10: 12: 03\end{array}$ & 22 & 0 \\
\hline 2 & ZY3 & $\begin{array}{c}05 / 13 \\
15: 31: 04\end{array}$ & 16 & 25 \\
\hline 5 & $\begin{array}{c}\text { ZY1- } \\
02 \mathrm{C}\end{array}$ & $\begin{array}{c}05 / 14 \\
12: 43: 45\end{array}$ & 15 & -20 \\
\hline 6 & $\begin{array}{c}\text { ZY1- } \\
02 \mathrm{C}\end{array}$ & $\begin{array}{c}05 / 15 \\
12: 11: 12\end{array}$ & 29 & 25 \\
\hline
\end{tabular}

Table 3. The schedule $S_{2}^{*}$

\begin{tabular}{|c|c|c|c|c|}
\hline $\begin{array}{c}\text { Meta } \\
\text { task }\end{array}$ & $\begin{array}{c}\text { Assigned } \\
\text { satellite }\end{array}$ & Start time & Duration/s & $\begin{array}{c}\text { Slewing } \\
\text { angle }^{\circ}\end{array}$ \\
\hline 7 & $\begin{array}{c}\text { ZY1- } \\
02 \mathrm{C}\end{array}$ & $\begin{array}{c}05 / 14 \\
12: 43: 22\end{array}$ & 31 & -25 \\
\hline
\end{tabular}

Table 4. The schedule $S_{3}^{*}$

When the earthquake breaks out, there is an urgent need to acquire the information about stricken area as soon as possible. The first imaging requirement is to acquire the information of 9 degree seismic intensity region. We use the GC-DAHA 
algorithm to obtain the first schedule $S_{1}^{*}$, as shown in Table 2 . From Figure 5(a) and Table 2, we can observe that the area task $r a_{1}$ is decomposed into four meta-tasks which are assigned to satellites and execution periods. The region of task $r a_{1}$ is entirely covered by observation strips. After collecting a portion of disaster information, the user puts forward new imaging requirement to observe Wenchuan County, namely $\mathrm{ra}_{2}$. Hence, this triggers the second dynamic scheduling. We insert new emergent task to $S_{1}^{*}$ and the second schedule $S_{2}^{*}$ is produced, as depicted in Figure 5(b) and Table 3. The result indicates that two meta-tasks are adjusted to satisfy new emergent requirement. As more disaster information has been obtained, the user submits a new imaging requirement to observe $r a_{3}$, which is the worst-hit region. Then dynamic scheduling is triggered again. The new schedule $S_{3}^{*}$ is shown in Figure 5(c) and Table 4. From the result, it is found that two meta-tasks have been performed observation until the third scheduling time. So there remain two meta-tasks in the waiting queue. The new task $r a_{3}$ are arranged in the third schedule $S_{3}^{*}$ by cancelling the two conflict tasks.

Besides, the expected reward of each schedule and the disturbance to the previous schedule are shown in Table 5. The disturbance is defined as total adjusted meta-tasks in each schedule. The results prove that the GC-DAHA algorithm can produce a schedule with high reward and small disturbances as far as possible.

\begin{tabular}{|c|c|c|}
\hline Schedule & Accumulated reward & Disturbance \\
\hline$S_{1}^{*}$ & 10 & 0 \\
\hline$S_{2}^{*}$ & 22 & 2 \\
\hline$S_{3}^{*}$ & 35.09 & 1 \\
\hline
\end{tabular}

Table 5. The reward and disturbance of schedules

\section{CONCLOSION}

To address the scheduling problem oriented to the dynamic areal tasks triggered by emergent disasters, we build a mathematical model in which the reward of the schedule is maximized. The dynamic segmenting algorithm is designed to partition area targets. Then we employ the GC-DAHA algorithm to obtain the optimal solution. To evaluate our model, we conduct experimental simulations in the scene of Wenchuan Earthquake. The simulated imaging plan can schedule satellites to observe a wide scope of target area. We conclude that our satellite scheduling model can optimize the usage of satellite resources so as to obtain images in disaster response in a more timely and efficient manner.

\section{ACKNOWLEDGEMENTS}

This work is partly supported by the National Basic Research Program of China (No. 2011CB707102), the National Natural Science Foundation of China (No. 41571334) and the Fundamental Research Funds for the Central Universities.

\section{REFERENCES}

Bai, B., Chen, Y., He, R., and Ruan, Q., 2009. Multi-satellite scheduling Toward Spot and Polygon observing Request. Journal of Astronautics, 30(2), pp. 755-759.

Bensana, E., Verfaillie, G., Agnese, J. C., Bataille, N., and Blumstein, D., 1996. Exact \&inexact methods for daily management of earth observation satellite. Space Mission Operations and Ground Data Systems (SpaceOps' 96), pp. 507515.

Bianchessi, N., Cordeau, J. F., Desrosiers, J., Laporte, G., and Raymond, V., 2007. A heuristic for the multi-satellite, multiorbit and multi-user management of Earth observation satellites. European Journal of Operational Research, 177(2), pp. 750762.

Gabrel V. and Vanderpooten D., 2002. Enumeration and interactive selection of efficient paths in a multiple criteria graph for scheduling an earth observing satellite. European Journal of Operational Research, 139(3), pp. 533-542.

Hall, N. G., and Magazine, M. J., 1994. Maximizing the value of a space mission. European Journal of Operational Research, 78, pp. 224-241.

Lemaitre, M. and Verfaillie, G., 1997. Daily management of an earth observation satellite: comparison of ILOG Solver with dedicated algorithms for Valued Constraint Satisfaction Problems.

Lemaître, M., Verfaillie, G., Jouhaud, F., Lachiver, J. M., and Bataille, N., 2002. Selecting and scheduling observations of agile satellites. Aerospace Science and Technology, 6(5), pp. 367-381.

Li, J., Yao, F., Bai, B., and Ruan, Q., 2008. Cooperative mission planing problem for area targets observation by multi satellites. Science of surveying and mapping, 33(S1), pp. 54-56.

Li, J., Yao, F., Bai, B., and He, R., 2009. A decompositionbased algorithm for imaging satellite scheduling problem. In Information Engineering and Computer Science, 2009. ICIECS 2009. International Conference on, pp. 1-6.

Lin, W. C., Liao, D. Y., Liu, C. Y., and Lee, Y. Y., 2005. Daily imaging scheduling of an earth observation. Systems, Man and Cybernetics, Part A: Systems and Humans, IEEE Transactions on, 35(2), pp. 213-223.

Mancel, C. and Lopez, P., 2003. Complex optimization problems in space systems. In 13th International Conference on Automated Planning \& Scheduling (ICAPS'03).

Mansour, M. A. and Dessouky, M. M., 2010. A genetic algorithm approach for solving the daily photograph selection problem of the SPOT5 satellite. Computers \& Industrial Engineering, 58(3), pp. 509-520.

Niu, X., Tang, H., Wu, L., Deng, R., and Zhai, X., 2015. Imaging-duration embedded dynamic scheduling of Earth observation satellites for emergent events. Mathematical Problems in Engineering, 2015.

Pemberton, J. C., 2000. Towards scheduling over-constrained remote sensing satellite. Proceedings of the $2 d$ International Workshop on Planning and Scheduling for Space. 
Ruan, Q., Tan, Y., Li, J., and Chen, Y., 2006. Research on segmenting and Selecting for area targets. Science of surveying and mapping, 31(1), pp. 98-100.

Walton, J. T., 1993. Models for the management of Satellitebased sensors (Doctoral dissertation, Massachusetts Institute of Technology).

Wang, J., Jing, N., Li, J., and Chen, Z. H., 2008. A multi objective imaging scheduling approach for earth observing satellites. Proceedings of the 9th Annual Conference on Genetic and Evolutionary Computation, 36(9), pp. 1715-1722.

Wang, P., Reinelt, G., Gao, P., and Tan, Y., 2011. A model, a heuristic and a decision support system to solve the scheduling problem of an earth observing satellite constellation. Computers \& Industrial Engineering, 61(2), pp. 322-335.

Wu, G., Liu, J., Ma, M., and Qiu, D., 2013. A two-phase scheduling method with the consideration of task clustering for earth observing satellites. Computers \& Operations Research, 40(7), pp. 1884-1894.

Zhai, X., Niu, X., Tang, H., Wu, L., and Shen, Y., 2015. Robust Satellite Scheduling Approach for Dynamic Emergency Tasks. Mathematical Problems in Engineering, 2015.

Zhang, Z., Zhang, N., and Feng, Z., 2014. Multi-satellite control resource scheduling based on ant colony optimization. Expert Systems with Applications, 41(6), pp. 2816-2823. 